\section{Antibiotic Prophylaxis and Postoperative Wound Infection Rates in Salvage Surgery for Head and Neck Cancer}

\section{Abstract}

Wound infections are the most common complication after free flap reconstruction of head and neck defects. Patients who have undergone prior chemo radiation have higher infection rates following surgery. We hypothesized that patients who undergo salvage surgery for recurrent or persistent head and neck cancer may benefit from prolonged antibiotic prophylaxis. The objectives are to determine the rate of infection in salvage surgery following chemo radiation and to determine type and duration of antibiotic prophylaxis. Retrospective review of patients with recurrent or persistent head and neck cancer who underwent clean-contaminated procedures with violation of the aerodigestive tract mucosa following radiation therapy or chemoradiation between 2010 to 2015 at Loyola University Medical Center. All patients underwent immediate reconstruction using a free flap or regional flap. Univariate analysis was performed. Outcomes measured included duration and type of antibiotic prophylaxis, development of postoperative surgical site infections, and common cultured pathogens. Sixty-six patients underwent salvage surgery with free flap reconstruction of which, 59 patients received antibiotic prophylaxis for more than 24 hours, 6 patients received it for less than 24 hours or less, and 1 patient received no antibiotic prophylaxis. The most common prophylactic antibiotics choices were clindamycin (38\%) and ampicillinsulbactam (26\%). Surgical site infections occurred in $56 \%(\mathrm{~N}=36)$ of patients, with mucocutaneous fistula as the most common (47.5\%). Staphylococcus aureus and Pseudomonas aeruginosa were the most commonly cultured organisms. Eightysix percent of patients receiving perioperative antibiotics for less than 24 hours developed a wound infection compared to $51 \%$ of patients receiving antibiotics for greater than 24 hours $(p=0.08)$. There is no benefit to extending antibiotic prophylaxis in patients undergoing salvage surgery beyond 24 hours.

Keywords: Surgical site infection; Antibiotic prophylaxis; Free flap; Microvascular reconstruction; Head and neck cancer; Salvage surgery; Chemo radiation

\section{Yang SF, Nadimi S, Eggerstedt M, Thorpe E and Pittman A}

Department of Otolaryngology, Loyola University Medical Center, Maywood, IL 60153, USA

\section{Corresponding Author: Yang SF}

\section{” shyang@lumc.edu}

Department of Otolaryngology, Loyola University Medical Center, 2160 S 1st Avenue, Maywood, IL 60153, USA.

\section{Tel: 7082165120}

Citation: Yang SF, Nadimi S, Eggerstedt M, et al. Antibiotic Prophylaxis and Postoperative Wound Infection Rates in Salvage Surgery for Head and Neck Cancer. Head Neck Cancer Res. 2016, 1:1.

\section{Introduction}

The importance of antibiotic prophylaxis in head and neck procedures is well established. Wound infection rates have been shown to be as high as $80 \%$ without antibiotic prophylaxis [1]. However, there is currently no Clinical Practice Guideline published by the American Academy of Otolaryngology- Head and Neck Surgery regarding antibiotic prophylaxis in head and neck surgery for cases contaminated by oropharyngeal secretions. Our current practice is thus governed by previously published data and also by the surgeon's anecdotal experience [1,2]. Previous studies have showed that 24 hours is sufficient in major head and neck cases [3-5].

Since the 1980s and 90s, when the first recommendations of antibiotic prophylaxis were published, there have been advances in the reconstructive techniques to regularly employ free flaps as opposed to regional flaps or primary closure in the reconstruction of major head and neck cases. We know that despite reconstructive advances, infections rates have not been positively impacted $[3,6,7]$. This may in part be due to known outside risk factors that impact infection rates. Specifically, in 
the setting of salvage surgery, in patients who have been treated previously with radiation therapy (RT) or chemoradiation (CR), there is an associated increased risk of surgical site infection [810]. In cases of salvage surgery and reconstruction, perhaps there lies a previously unidentified patient subset that might benefit from prolonged antibiotic prophylaxis. The purpose of this study was to determine if patients who underwent prior RT or CR were at increased risk for postoperative wound infection and if they would benefit from antibiotic prophylaxis longer than 24 hours. To our knowledge, no study has been performed to determine optimal timing and selection of antibiotic prophylaxis in patients who have previously undergone RT or CR of the recipient surgical site.

\section{Methods}

After obtaining approval from the Institutional Review Board at the Loyola University Medical Center (protocol \#206970), a retrospective chart review was performed of all adult patients (>18 years old) who underwent salvage surgery for head and neck malignancy with immediate free flap or regional flap reconstruction. Salvage surgery included all clean-contaminated ablative procedures where the mucosa of the aerodigestive tract was entered after failed previous RT or CR. All surgeries were performed at Loyola University Medical Center between $1 / 1 / 2010$ and $6 / 31 / 2015$ and were performed by 3 surgeons. ICD-9 codes were queried to identify patients who underwent ablative procedures with immediate free flap, gastric pull up, latissimus dorsi flap, deltopectoral flap, or pectoralis major flap reconstruction for recurrent or persistent head and neck malignancy. Patients with no prior radiation and/or chemotherapy were excluded. Data including sex, body mass index, preoperative albumin, history of tobacco and alcohol use, diabetes, positive surgical margins, immediate tracheoesophageal puncture, preoperative MRSA nasal swab, type of procedure, previous chemotherapy, previous radiation therapy, and type and duration of antibiotic prophylaxis were obtained. Patients were divided into two groups based on duration of antibiotic prophylaxis that was given based on surgeon preference. Those patients who received 24 hours or less of antibiotic prophylaxis were included in the short-term group and those patients who received antibiotic prophylaxis for longer than 24 hours were included in the long-term group. The primary outcome was incidence of surgical site infection measured from postoperative day one to date of last follow-up, which ranged from postoperative day 30 to postoperative day 90 . Surgical site infection was defined clinically as fistula formation with or without purulent drainage, cellulitis (presence of severe erythema or edema), and abscess formation at the recipient surgical site. Univariate analysis was performed for data analysis. All statistical calculations were performed using SPSS version 20 statistical software with significance defined as $\mathrm{P}<0.05$.

\section{Results}

Sixty-six patients underwent salvage surgery for persistent or recurrent head and neck cancer. Of the 66 patients, 49 (74\%) were male and 17 (26\%) were female. Fifty-five (83\%) patients were current or former smokers, $12(18 \%)$ were diabetic, 36
(54\%) reported past or current use of alcohol, and $14(21 \%)$ had a BMI of less than 20 and 17 (26\%) had a BMI of greater than 25. See Table 1 for complete patient characteristics. The majority of patients were previously treated with CR (51\%). Forty-seven percent of patients were previously treated with RT alone. The most common time between salvage surgery and prior treatment being less than 6 months or greater than 5 years (Figure 1). Patients underwent salvage surgery consisting of composite resection of the mandible (44\%), total laryngopharyngectomy (21\%), total laryngectomy (15\%), midline mandibulotomy with total glossectomy (4.5\%), and hemiglossectomy (4.5\%). The types of free flaps used for reconstruction included fibula free flap (35\%), radial forearm free flap (35\%), anterolateral thigh free flap (25\%), and rectus abdominis free flap (4\%). Regional flap reconstruction included pectoralis major (76\%), latissimus dorsi $(8 \%)$, deltopectoral $(8 \%)$, and gastric pullup (8\%).

Fifty-nine patients received antibiotic prophylaxis for more than 24 hours, six patients received it for less than 24 hours or less, and one patient received no antibiotic prophylaxis. Of the 66 patients included, six (86\%) of the seven patients who received antibiotic prophylaxis for less than 24 hours developed a wound complication, whereas $30(51 \%)$ of the 59 patients who received antibiotics for greater than 24 hours had complications $(p=0.08)$ (Figure 1). Thirty-six (56\%) patients developed a surgical site

Table 1 Demographic data of patients with prior history of radiation and/or chemotherapy that underwent salvage surgery and free flap reconstruction.

\begin{tabular}{|c|c|}
\hline Characteristic & \# of Patients (\%) \\
\hline Sex & \\
\hline Male & $49(74 \%)$ \\
\hline Female & $17(26 \%)$ \\
\hline BMI & \\
\hline$<20$ & $14(21 \%)$ \\
\hline $20-25$ & $35(53 \%)$ \\
\hline$>25$ & $17(26 \%)$ \\
\hline Diabetes & $12(18 \%)$ \\
\hline Past or Current Smoker & $66(83 \%)$ \\
\hline Past or Current Alcohol Use & $36(54 \%)$ \\
\hline Positive MRSA nasal swab & $3(4.5 \%)$ \\
\hline Positive Surgical Margins & $24(36 \%)$ \\
\hline
\end{tabular}

Antibiotics $\leq 24 \mathrm{hr}$ Antibiotics $>24 \mathrm{hr}$
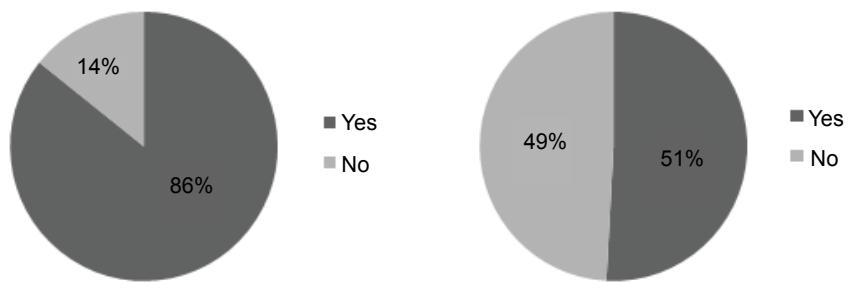

Figure 1 Percentage of patients with prior history of radiation and/or chemotherapy who underwent salvage surgery and free flap reconstruction who developed wound complication based on duration of antibiotic prophylaxis $(p=0.08)$. 
infection, which included mucocutaneous fistula (47.5\%), cellulitis (37.5\%), and abscess (15\%). Four patients (6\%) developed more than one complication. Sixty-one percent of complications occurred within the first two weeks of surgery.

Clindamycin (38\%) and ampicillin-sulbactam (26\%) were the most common antibiotics used for prophylaxis. Duration of antibiotic prophylaxis ranged from 0 days to greater than 5 days with the most common duration 3-4 days (26\%). Staphylococcus aureus and Pseudomonas aeruginosa were the most commonly cultured organisms. Gentamicin, tobramycin, meropenem, and sulfamethoxazole-trimethoprim were the most sensitive against the cultured organisms (Tables $\mathbf{2}$ and $\mathbf{3}$ ).

\section{Discussion}

Postoperative surgical site infection is not uncommon following salvage surgery for head and neck malignancy. Previous studies have cited infection rates up to $61 \%[10,11]$. Our findings are consistent with this as surgical site infection occurred in more than half $(54.5 \%)$ of our patients undergoing salvage surgery. There have been many studies that have analyzed different patient risk factors and their potential role as a predictor in the development of surgical site infections. Risk factors analyzed

Table 2 Type and number of organisms cultured from patients with postoperative wound infections after $\mathrm{H} \& \mathrm{~N}$ reconstruction.

\begin{tabular}{|c|c|}
\hline Organism Cultured & Number \\
\hline Staphylococcus aureus & 8 \\
\hline Pseudomonas aeruginosa & 6 \\
\hline Stretococcus & 4 \\
\hline Escherichia coli & 3 \\
\hline Klebsiella pneumoniae & 3 \\
\hline Enterococcus & 2 \\
\hline Methicillin-resistant Staphylococcus aureus & 1 \\
\hline Enterobacter aerogenes & 1 \\
\hline Corynebacterium & 1 \\
\hline Peptostreptococcus & 1 \\
\hline Prevotella Melaningenica & 1 \\
\hline
\end{tabular}

Table 3 Antibiotic sensitivities of cultures from patients who developed postoperative wound infections.

\begin{tabular}{|c|c|}
\hline Antibiotic & Percent of Cultures Sensitive (\%) \\
\hline Gentamycin & 14 \\
\hline Tobramycin & 11 \\
\hline Meropenam & 11 \\
\hline Cefepime & 10 \\
\hline Ceftazidine & 10 \\
\hline Ciprofloxacin & 10 \\
\hline Piperacillin-Tazobactam & 10 \\
\hline Bactrim & 11 \\
\hline Aztreonam & 9 \\
\hline Piperacillin & 9 \\
\hline Ceftriaxone & 8 \\
\hline Flagyl & 5 \\
\hline Cefazolin & 7 \\
\hline Levaquin & 5 \\
\hline Unasyn & 6 \\
\hline
\end{tabular}

Table 4 Comparison of incidence of wound complication based on duration of antibiotic prophylaxis $(p=0.08)$.

\begin{tabular}{|c|c|c|c|}
\hline & $\begin{array}{c}\mathbf{5 2 4} \mathbf{~ h r} \\
\text { Prophylaxis }\end{array}$ & $\begin{array}{c}\mathbf{> 2 4} \mathbf{~ h r} \\
\text { Prophylaxis }\end{array}$ & Total \\
\hline $\begin{array}{c}\text { Wound } \\
\text { Complication }\end{array}$ & $6(86 \%)$ & $30(51 \%)$ & 36 \\
\hline $\begin{array}{c}\text { No Wound } \\
\text { Complication }\end{array}$ & $1(14 \%)$ & $29(49 \%)$ & 30 \\
\hline Total & 7 & 59 & 66 \\
\hline
\end{tabular}

include age, tobacco use, prior radiation, length of hospital stay, tumor stage, tracheotomy, and malnutrition [6-8,10,12]. There is conflicting data on the association between prior RT and postoperative surgical site infection [9,13-16]. A retrospective cohort study of 698 patients revealed that preoperative RT was the strongest predictor of wound complications on both univariate and multivariate analysis [13]. Another study of 429 patients reported previous radiotherapy at any dose as a risk factor for fistula formation and surgical site infection; and doses $>60$ Gy was predictive of flap failure [9]. Recently, Yarlagadda et al. [8] reported that patients who had undergone prior RT displayed almost twice the rate of surgical site infection after ablative surgery and free flap reconstruction for head and neck cancer (Table 4). Despite these findings, other previous studies have not been able to demonstrate a significant correlation between prior radiation and flap-related complications or flap failure [14-16].

The difficulty in the post-operative treatment of patients who undergo clean-contaminated procedures lies in the development of post-operative fistula, which was classified in this study as a post-operative infection. In this study, the most common surgical site infection was mucocutaneous fistula followed by cellulitis and then abscess. It could be extrapolated that these infections are really a spectrum of severity, which often start with fistula formation. Of the patients who had post-operative infections, almost two-thirds of the complications were detected within the first 14 days following surgery and $42 \%$ required surgical intervention. These complications can significantly delay postoperative healing, increase morbidity, and prolong hospital length of stay [17-19].

Given the consequences of surgical site infection, prevention is important and there may be benefit to stratify patients who are at increased risk for infection. The clinical question as to whether salvage surgical patients should be treated as higher risk and given longer duration of antibiotic prophylaxis is a valid one. Although prior studies have found no difference in wound infection rates when comparing shorter course antibiotic therapy (24 hours) to longer duration antibiotic therapy ( $>24$ hours), these studies did not stratify patients who received prior RT or CR. To our knowledge, there is no data in the literature on whether certain higher-risk groups may benefit from longer-duration antibiotic therapy. In this study, we found that 6 of the 7 patients (86\%) with a history of radiation +/- chemotherapy who received antibiotic therapy for 24 hours or less developed a wound complication compared to 30 of 59 patients (51\%) who received antibiotic therapy for greater than 24 hours. This finding suggests that prolonged antibiotic prophylaxis ( $>24$ hours) is associated with decreased incidence of wound complication. This difference, however, did 
not reach statistical significance $(p=0.08)$, and thus justification for prolonging antibiotics in salvage cases cannot be made based on the evidence. Unfortunately, one of the drawbacks of this study is that the group who actually received antibiotics for less than or equal to 24 hours was much smaller than the longer term group [20-23].

Certainly the trend in this institution was to continue long-term antibiotic prophylaxis. We are not alone in that practice. One study showed that many reconstructive surgeons continue to use prophylactic antibiotics for longer than 72 hours following free flap reconstruction $[12,17]$ which likely is based on anecdotal evidence or perseverance in practices which have been in place previously. This observation led us to question antibiotic prophylaxis selection and also bacterial speciation of cultured infections.

Clindamycin and ampicillin-sulbactam are two commonly used antibiotics for perioperative prophylaxis $[12,18]$ and were the most commonly used antibiotics for prophylaxis in our study. Wound cultures obtained from postoperative complication sites most commonly grew Staphylococcus aureus and Pseudomonas aeruginosa and $38 \%$ of cultures were polymicrobial. Only $40 \%$ of cultures were sensitive to clindamycin and ampicillin-sulbactam. These results put into question the appropriate selection of antibiotics. There have been studies that demonstrated an increased risk of postoperative wound infection with the use of clindamycin $[12,18]$.

Another important question is if inappropriate duration of antibiotic prophylaxis perpetuates bacterial resistance resulting in increased susceptibility to wound infection. One study assessed the association between extended duration of antibiotic prophylaxis and risk of postoperative infection. They did not find an increased risk of infection with antibiotic prophylaxis of 7-8 days compared to 1 day $[12,24]$. Another study looked at the onset of surgical site infection and the associated microbiology. Three fourths of cultures from this study were polymicrobial and grew pathogens not part of normal flora. Furthermore, $67 \%$ of cultures demonstrated resistance to prophylactic antibiotics used. There was no difference in type of pathogen cultured based on the when the surgical site infection occurred [25]. Given these findings and increased antibiotic resistance patterns, appropriate selection of antibiotic prophylaxis is crucial.

This study has its limitations. Given this study is retrospective, the data is limited to the medical records. In addition, the study involved three surgeons performing the reconstruction, which may have influenced the type and duration of antibiotics used postoperatively. Furthermore, the size of the two study groups were not equal as the longer than 24 hour antibiotic prophylaxis group was larger than the group that received prophylaxis for less than 24 hours. With the small sample size there was not enough power to reach statistical significance. This study could be improved using a multivariate analysis to control for other factors such as medical comorbidities, social history, preoperative nutritional status, and positive surgical margins, which may contribute to development of wound complications and confound the findings of this study.

\section{Conclusion}

Postoperative wound complication rates following salvage surgery remain high. There have been data to suggest that patients who have had prior RT or CR may be at increased risk for postoperative wound complications and may benefit from prolonged antibiotic treatment. This study did not demonstrate a benefit in the use of long-term antibiotic prophylaxis in this patient population. Additionally, a majority of postoperative wound infections were resistant to clindamycin and ampicillin-sulbactam, which are commonly used for prophylaxis. This questions whether these are the best antibiotic choices for salvage surgery cases, and if the practice of long term antibiotic prophylaxis contributes to bacterial resistance. 


\section{References}

1 Johnson JT, Yu VL (1988) Antibiotic use during major head and neck surgery. Ann Surg 207: 108-111.

2 Johnson JT, Wagner RL, Schuller DE, Gluckman J, Suen JY, et al. (1992) Prophylactic antibiotics for head and neck surgery with flap reconstruction. Arch Otolaryngol Head Neck Surg 118: 488-490.

3 Carroll WR, Rosenstiel D, Fix JR, de la Torre J, Solomon JS, et al. (2003) Three-dose vs extended-course clindamycin prophylaxis for free-flap reconstruction of the head and neck. Arch Otolaryngol Head Neck Surg 129: 771-774.

4 Mitchell RM, Mendez E, Schmitt NC, Bhrany AD, Futran ND (2015) Antibiotic Prophylaxis in Patients Undergoing Head and Neck Free Flap Reconstruction. JAMA Otolaryngol Head Neck Surg 141: 1096-1103.

5 Johnson JT, Schuller DE, Silver F, Gluckman JL, Newman RK, et al. (1986) Antibiotic prophylaxis in high-risk head and neck surgery: one-day vs. five-day therapy. Otolaryngol Head Neck Surg 95: 554-557.

6 Girod DA, McCulloch TM, Tsue TT, Weymuller EA Jr (1995) Risk factors for complications in clean-contaminated head and neck surgical procedures. Head Neck 17: 7-13.

7 Robbins KT, Favrot S, Hanna D, Cole R (1990) Risk of wound infection in patients with head and neck cancer. Head Neck 12: 143-148.

8 Yarlagadda BB, Deschler DG, Rich DL, Lin DT, Emerick KS, et al. (2016) Head and neck free flap surgical site infections in the era of the Surgical Care Improvement Project. Head Neck 38 Suppl 1: E392-E398.

9 Benatar MJ, Dassonville O, Chamorey E, Poissonnet G, Ettaiche M, et al. (2013) Impact of preoperative radiotherapy on head and neck free flap reconstruction: a report on 429 cases. J Plast Reconstr Aesthet Surg 66: 478-482.

10 Penel N, Lefebvre D, Fournier C, Sarini J, Kara A, et al. (2001) Risk factors for wound infection in head and neck cancer surgery: a prospective study. Head Neck 23: 447-455.

11 Scotton W, Cobb R, Pang L, Nixon I, Joshi A, et al. (2012) Post-operative wound infection in salvage laryngectomy: does antibiotic prophylaxis have an impact? Eur Arch Otorhinolaryngol 269: 2415-2422.

12 Mitchell RM, Mendez E, Schmitt NC, Bhrany AD, Futran ND (2015) Antibiotic Prophylaxis in Patients Undergoing Head and Neck Free Flap Reconstruction. JAMA Otolaryngol Head Neck Surg 141: 1096-1103.

13 Disa JJ, Pusic AL, Hidalgo DH, Cordeiro PG (2001) Simplifying microvascular head and neck reconstruction: a rational approach to donor site selection. Ann Plast Surg 47: 385-389.
14 Tan NC, Lin PY, Chiang YC, Chew KY, Chen CC, et al. (2014) Influence of neck dissection and preoperative irradiation on microvascular head and neck reconstruction-Analysis of 853 cases. Microsurgery 34: 602-607.

15 Lin S, Dutra J, Keni J, Dumanian GA, Fine N, et al. (2005) Preoperative radiation therapy and its effects on outcomes in microsurgical head and neck reconstruction. Otolaryngol Head Neck Surg 132: 845-848.

16 Haughey BH, Wilson E, Kluwe L, Piccirillo J, Fredrickson J, et al. (2001) Free flap reconstruction of the head and neck: analysis of 241 cases. Otolaryngol Head Neck Surg 125: 10-17.

17 Reiffel AJ, Kamdar MR, Kadouch DJ, Rohde CH, Spector JA (2010) Perioperative antibiotics in the setting of microvascular free tissue transfer: current practices. J Reconstr Microsurg 26: 401-407.

18 Durand ML, Yarlagadda BB, Rich DL, Lin DT, Emerick KS, et al. (2015) The time course and microbiology of surgical site infections after head and neck free flap surgery. Laryngoscope 125: 1084-1089.

19 Bratzler DW, Dellinger EP (2013) American Society of Health-System Pharmacists; Infectious Diseases Society of America; Surgical Infection Society; Society for Healthcare Epidemiology of America. Clinical practice guidelines for antimicrobial prophylaxis in surgery. Surg Infect (Larchmt) 14: 73-156.

20 Kucur C, Durmus K, Uysal IO (2016) Management of complications and compromised free flaps following major head and neck surgery. Eur Arch Otorhinolaryngol 273: 209-213.

21 Cohen LE, Finnerty BM, Golas AR, Ketner JJ, Weinstein A, et al. (2016) Perioperative Antibiotics in the Setting of Oropharyngeal Reconstruction: Less is More. Ann Plast Surg 76: 663-667.

22 De Wilde RL, Donders G (1986) Scanning electron microscopic study of microvascular anastomoses on irradiated vessels: long-term effect of irradiation. Microsurgery 7: 156-157.

23 Guelinckx PJ, Boeckx WD, Fossion E, Gruwez JA (1984) Scanning electron microscopy of irradiated recipient blood vessels in head and neck free flaps. Plast Reconstr Surg 74: 217-226.

24 Watson JS (1979) Experimental microvascular anastomoses in radiated vessels: a study of the patency rate and the histopathology of healing. Plast Reconstr Surg 63: 525-533.

25 Schultze-Mosgau S, Grabenbauer GG, Radespiel-Tröger M, Wiltfang $\mathrm{J}$, Ries J, et al. (2002) Vascularization in the transition area between free grafted soft tissues and pre-irradiated graft bed tissues following preoperative radiotherapy in the head and neck region. Head Neck 24: 42-51. 